\title{
Nutcracker Syndrome due to Chronic Aortic Dissection
}

\author{
YUKI ICHIHARA ${ }^{1}$, Takashi Azuma ${ }^{1}$, Satoshi Saito ${ }^{1}$, and Hiroshi Niinami ${ }^{1}$ \\ ${ }^{1}$ Tokyo Women's Medical University
}

November 24, 2020

\begin{abstract}
Nutcracker syndrome (NCS) is known as a status of compression of the left renal vein (LRV) between the abdominal aorta and superior mesenteric artery (SMA). We here report a case of NCS in Marfan patients with type B aortic dissection who presented with sudden gross hematuria. Computed tomography revealed the compression of the LRV sandwiched between the SMA and the dilated dissecting abdominal aorta. The compression was released after surgical intervention and the hematuria was promptly recovered. This report highlights that NCS should be considered as a differential diagnosis of unexplained hematuria in patients with the chronic dissecting aorta.
\end{abstract}

\section{Nutcracker Syndrome due to Chronic Aortic Dissection}

Yuki Ichihara, $\mathrm{MD}, \mathrm{PhD}^{+}$, Takashi Azuma, MD, PhD, Satoshi Saito, MD, PhD,

Hiroshi Niinami, MD, PhD

Department of Cardiovascular Surgery, Tokyo Women's Medical University

+ Corresponding author: Yuki Ichihara

8-1, Kawada-cho, Shinjuku-ku, Tokyo, Japan, 162-8666

Tel:(+81) 3-3353-8111

Fax:(+81) 3-3356-0441

Email:ichihara.yuki@twmu.ac.jp

Word count:500/500

\section{Abstract}

Nutcracker syndrome (NCS) is known as a status of compression of the left renal vein (LRV) between the abdominal aorta and superior mesenteric artery (SMA). We here report a case of NCS in Marfan patients with type B aortic dissection who presented with sudden gross hematuria. Computed tomography revealed the compression of the LRV sandwiched between the SMA and the dilated dissecting abdominal aorta. The compression was released after surgical intervention and the hematuria was promptly recovered. This report highlights that NCS should be considered as a differential diagnosis of unexplained hematuria in patients with the chronic dissecting aorta.

Text

Nutcracker syndrome (NCS) is caused by compression of the left renal vein (LRV) between the abdominal aorta and superior mesenteric artery (SMA), resulting in several urological symptoms ${ }^{[1]}$.

A 36-year-old male with Marfan-like features, who had developed type B aortic dissection 3 months prior, presented with sudden gross hematuria. Computer tomography (CT) revealed obstruction of the LRV 
sandwiched between the dissecting aneurysm of abdominal aorta and the SMA, so-called "nutcracker phenomenon" (A ). Abrupt narrowing of the LRV at the aortomesenteric (AM) portion (beak sign) (B ) as well as the great ratios of the diameter of the LRV between at the AM portion (2.1mm) and the hilar portion $(11.8 \mathrm{~mm})$ were identified, resulting in a diagnosis of $\mathrm{NCS}^{[2]}$. Gross hematuria remained persistent, thus our team decided to perform surgical intervention. Obstruction of the LRV was released after the graft replacement of thoracoabdominal aorta $(\mathbf{C})$ and hematuria was promptly relieved. Three-dimensional CT illustrated the preoperative abnormally acute angle between the SMA and aorta (D, 17 degrees) was clearly widened (E , 40 degrees) after the operation.

This is the first report of NCS caused by a dissecting aneurysm of the thoracoabdominal aorta and its successful surgical management. NCS should be considered as a differential diagnosis for unexplained hematuria in patients with chronic aortic dissection.

\section{Disclosure}

The authors declare no conflicts of interest.

\section{References}

De Schepper A. "Nutcracker" phenomenon of the renal vein causing left renal vein pathology. J Belge Radiol 1972;55(5):507-511.

Kim KW, Cho JY, Kim SH, Yoon JH, Kim DS, Chung JW, et al . Diagnostic value of computed tomographic findings of nutcracker syndrome: Correlation with renal venography and renocaval pressure gradients. Eur J Radiol 2011;80(3):648-654.

\section{Figure legend}

(A ) Preoperative computed tomography (CT) image showing compression of the left renal vein (LRV, arrowhead) between the dilated abdominal aorta (AO) and superior mesenteric artery (SMA). (B ) Abrupt triangular narrowing of the LRV (beak sign). (C) Postoperative CT image showing distance between the prosthetic graft (PG) and SMA, as well as the release of compression affecting the LRV. (D, E ) Preand postoperative three-dimensional CT images showing enlargement of the angle between the SMA and aorta/PG.
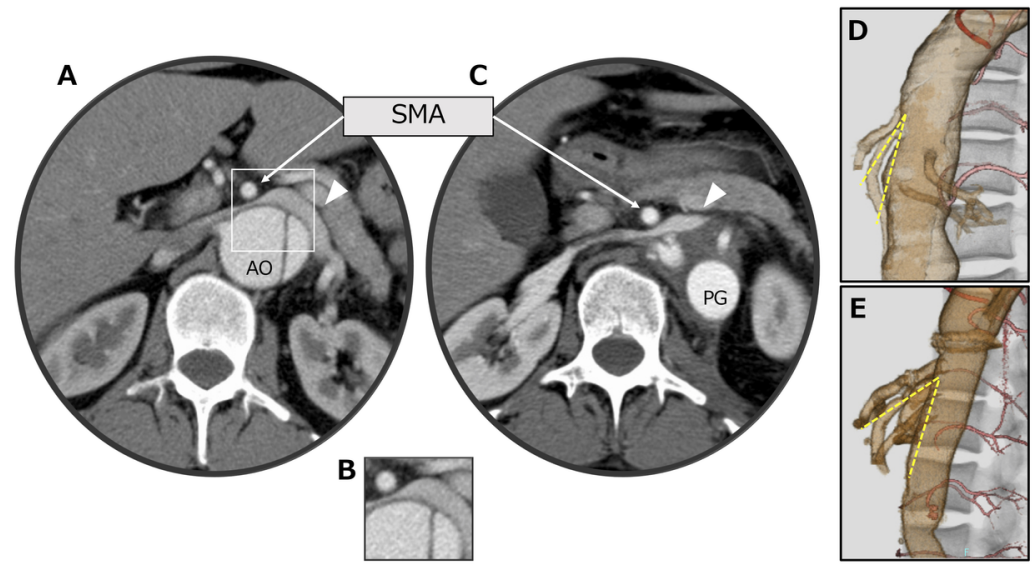